\title{
Using metal-organic templates to steer the growth of Fe and Co nanoclusters
}

\author{
R. Decker, ${ }^{1}$ U. Schlickum, ${ }^{1}$ F. Klappenberger, ${ }^{2}$ G. Zoppellaro, ${ }^{3}$ S. Klyatskaya, ${ }^{3}$ \\ M. Ruben, ${ }^{3}$ J. V. Barth, ${ }^{2}$ and H. Brune ${ }^{1, a)}$ \\ ${ }^{1}$ Institute of the Physics of Nanostructures, Ecole Polytechnique Fédérale de Lausanne (EPFL), CH-1015 \\ Lausanne, Switzerland \\ ${ }^{2}$ Department of Physics E20, Technische Universität München, D-85748 Garching, Germany \\ ${ }^{3}$ Institute of Nanotechnology, Forschungszentrum Karlsruhe, D-76021 Karlsruhe, Germany
}

(Received 11 July 2008; accepted 12 November 2008; published online 15 December 2008)

\begin{abstract}
Regularly sized Fe and Co nanostructures are created using two-dimensional metal-organic honeycomb lattices as templates. They consist of dicarbonitrile-polyphenyl molecules coordinated to Co centers on $\mathrm{Ag}(111)$. Subsequently deposited Fe or Co atoms nucleate clusters at specific sites on top of the metal-organic lattices while leaving their hexagonal pores empty. Choosing the deposition temperature, one can selectively decorate the molecules $(120 \mathrm{~K})$ or the Co coordination nodes (190 K) with Fe. The distance between the Fe and Co clusters and their shape can be adjusted through the lattice constant of the template by varying the number of phenyl rings in the molecules. (C) 2008 American Institute of Physics. [DOI: 10.1063/1.3040328]
\end{abstract}

Self-organized growth of metals on template surfaces is a promising route to create ensembles of nanostructures with well-defined sizes and a high degree of order over micrometer distances. Metallic nanostructures have attracted interest since they exhibit magnetic ${ }^{1}$ and catalytic properties ${ }^{2,3}$ that are distinctive from the ones of their bulk counterparts. These properties often show a strong size dependence, where every constituent atom counts. In addition, they are determined by the chemical environment of the nanostructures and their mutual distance. Therefore templates where these parameters can be varied are of high interest. So far, ordered arrays of nanostructures have been assembled on strain-relief patterns, ${ }^{4-7}$ on vicinal surfaces, ${ }^{8-10}$ and on ultrathin insulating layers on metals. ${ }^{11,12}$

We present a method for the self-organized growth of metal nanoclusters using metal-organic nanomeshes as templates. The advantages of our approach are the adjustable mutual distance and chemical environment of the metal clusters.

A large variety of ordered organic and metal-organic networks on surfaces has been studied during the past few years. ${ }^{13}$ It has been shown that these systems have great potential as templates for the ordered adsorption of organic molecules. ${ }^{14,15}$ However, examples of metal deposition on organic or metal-organic templates are rare. While layers of organic molecules have been demonstrated to steer nucleation of metal atoms toward the molecule covered step bunches of a Ag vicinal surface, ${ }^{16}$ regularly spaced and sized metal nanostructures grown on organic or metal-organic templates have to our knowledge so far not been reported.

We recently showed the fabrication of metal-organic honeycomb nanomeshes forming well ordered single domains extending over $\mu \mathrm{m}^{2}$ areas. ${ }^{17}$ The pore sizes are tunable via the length of the molecular building blocks from 10 to $20 \mathrm{~nm}^{2}$. In this letter we demonstrate the potential of these metal-organic lattices to serve as templates for the growth of regularly sized $\mathrm{Fe}$ and Co nanostructures. The dis-

${ }^{a)}$ Electronic mail: harald.brune@epfl.ch. tance between the nanostructures can be varied by changing the lattice constant of the template. In addition, their location on the network and therefore their chemical environment can be chosen by the deposition temperature.

The measurements were carried out in ultrahigh vacuum ( $p \leqslant 3 \times 10^{-10}$ mbar) using a home-built scanning tunneling microscope (STM) operating at $10 \mathrm{~K}^{18}$ The metal-organic templates were grown on a $\operatorname{Ag}(111)$ surface, prepared by repeated cycles of $1 \mathrm{keV} \mathrm{Ar}^{+}$sputtering at room temperature $\left(p_{\mathrm{Ar}^{+}}=10^{-6} \mathrm{mbar}, I=5 \mu \mathrm{A}\right)$ and subsequent annealing to $800 \mathrm{~K}$. Depending on the desired lattice constant of the template, we used one of three dicarbonitrile-polyphenyl molecules $\left(\mathrm{NC}-\mathrm{Ph}_{n}-\mathrm{CN}\right)$, containing $n \in\{3,4,5\}$ phenyl rings. ${ }^{19}$ The phenyl rings form linear chains terminated at both ends by carbonitrile groups. In the gas phase and in the planar configuration, the lengths of the molecules from one $\mathrm{N}$ atom to the other are $1.66,2.09$, and $2.51 \mathrm{~nm}$, respectively (HYPERCHEM calculations). They were evaporated by organic molecular beam epitaxy from a Knudsen cell at $460 \mathrm{~K}\left(\mathrm{NC}-\mathrm{Ph}_{3}-\mathrm{CN}\right), 490 \mathrm{~K}\left(\mathrm{NC}-\mathrm{Ph}_{4}-\mathrm{CN}\right)$, and $535 \mathrm{~K}$ $\left(\mathrm{NC}-\mathrm{Ph}_{5}-\mathrm{CN}\right)$ onto the $\mathrm{Ag}(111)$ surface kept at $300 \mathrm{~K}$. The molecules were then linked into the honeycomb lattices by depositing Co in stoichiometric amounts from an electron beam evaporator at a substrate temperature of $300 \mathrm{~K}$. Figure 1 displays a STM topograph of a honeycomb network together with a model showing the threefold Co coordination by $\mathrm{CN}$ groups. ${ }^{20}$ The unit cell has the stoichiometry $\left(\mathrm{NC}-\mathrm{Ph}_{n}-\mathrm{CN}\right)_{3} \mathrm{Co}_{2}$.

$\mathrm{Co}$ and $\mathrm{Fe}$ atoms were deposited onto the metal-organic honeycomb network at sample temperatures between 85 and $220 \mathrm{~K}$. Figure 2(a) shows the surface after deposition of Co onto $\left(\mathrm{NC}-\mathrm{Ph}_{3}-\mathrm{CN}\right)_{3} \mathrm{Co}_{2}$ at $116 \mathrm{~K}$. The Co clusters appear as bright protrusions in the STM image. The template remains unperturbed up to much higher Co coverage, showing its robustness toward the highly reactive Co atoms. The clusters have a mean size of $2.6 \pm 1.0$ atoms, as deduced from the Co coverage and the cluster density. Surprisingly, they form exclusively on top of the metal-organic template, more precisely, on the phenyl moieties of the organic molecules. This 


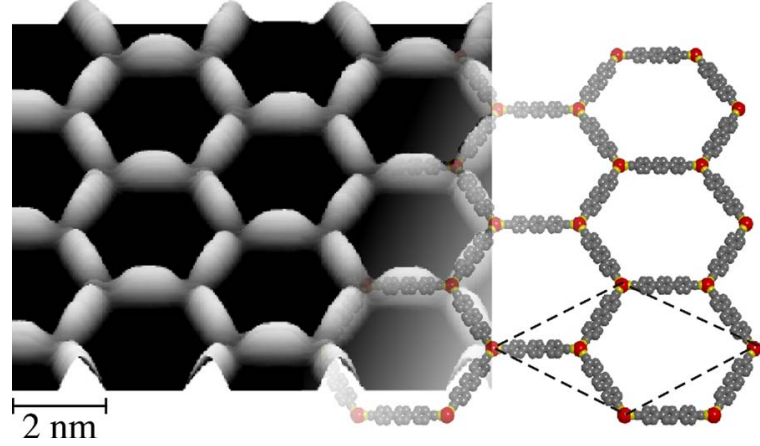

FIG. 1. (Color online) Constant current STM image of a honeycomb net-

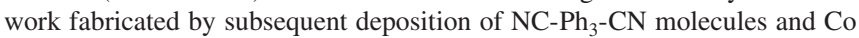
atoms on a $\mathrm{Ag}(111)$ surface. The molecular model is superimposed (orange, cobalt centers; gray, carbon; yellow, nitrogen). The dotted line illustrates the honeycomb unit cell [tunnel current $I_{t}=0.3 \mathrm{nA}$, tunnel voltage applied to sample $V_{t}=2.0 \mathrm{~V}$, and STM image processed with WSXM (Ref. 27)].

implies that Co atoms diffuse from the Ag substrate exposed in the honeycomb cavities toward and then onto the molecules. The mobility required for reaching the molecules is largely sufficient since Co deposition onto clean $\operatorname{Ag}(111)$ at identical temperature leads to islands approximately $50 \mathrm{~nm}$ apart, in agreement with the diffusion barrier and prefactor determined for that system. ${ }^{21}$ The on-top bonding of Co atoms on the phenyl rings of the organic ligands has been observed for deposition temperatures up to $250 \mathrm{~K}$ and can be rationalized by the formation of half-sandwich-type complexes. ${ }^{22}$ It is difficult to precisely determine the binding energy of a Co atom on a phenyl ring adsorbed onto $\mathrm{Ag}(111)$ in order to compare it with the one of Co on a $\operatorname{Ag}(111)$ terrace. The following estimate shows, however, that they are very close. The binding energy of Co on a benzene molecule in the gas phase has been measured to be $2.65 \pm 0.11 \mathrm{eV}$ (Ref. 23) and calculated to be $2.58 \mathrm{eV} .^{24}$ The adsorption energy of $\mathrm{Co} / \mathrm{Ag}(111)$ is expected to be slightly higher than the one for $\mathrm{Ag} / \mathrm{Ag}(111)$, which has been measured to be $2.6 \mathrm{eV}$ (Ref. 25) and calculated to be $2.8 \mathrm{eV} .^{26}$ It is likely that a phenyl ring lying flat on $\mathrm{Ag}(111)$ binds Co stronger than a benzene molecule in the gas phase, which would explain our observation.

$\mathrm{Fe}$ atoms assemble in a similar manner as Co on the metal-organic network, as seen in Fig. 2(b). Already at the low coverage in Fig. 2(b), there are locations where all molecules of one hexagon are iron decorated. This results in ring configurations, where neighboring Fe clusters are separated by the coordination nodes. From inspection of Fig. 2(b), the distribution of the apparent lateral cluster sizes is quite narrow. The histogram in Fig. 2(c) shows that also the apparent cluster height has a very narrow distribution with $190 \pm 15 \mathrm{pm}$. These two observations indicate that the apparent volume of the Fe clusters is very well defined. The mean cluster size is $2.6 \pm 1.6$ atoms, with the error bar reflecting the uncertainty in the coverage coming from the Fe flux calibration.

By varying the length of the molecular building blocks, we can change the pore size of our templates and thus the shape and spacing of the metal structures. Figure 3(a) shows an Fe cluster superlattice on a $\left(\mathrm{NC}-\mathrm{Ph}_{5}-\mathrm{CN}\right)_{3} \mathrm{Co}_{2}$ template for two times higher Fe coverage than that in Fig. 2(b). The coverage increase leads to the decoration of nearly every organic molecule and thus to the formation of a more regular arrangement of Fe clusters. The enhanced length of the phenyl chain shows the same nucleation behavior as the shorter one; however, it allows the formation of either larger, more elongated clusters or of two small clusters on one phenyl chain. Very similar results are obtained also for $\mathrm{Co}$, however, only for small coverage. The size uniformity is better for $\mathrm{Fe}$ than for Co, especially with increasing coverage, where in the case of Co large and randomly placed clusters appear.

In the case of Fe one can control the nucleation site by the deposition temperature. While clusters are located on the phenyl rings for $85 \mathrm{~K} \leqslant T_{\text {dep }} \leqslant 130 \mathrm{~K}$, nucleation takes place at the network nodes for $190 \mathrm{~K} \leqslant T_{\text {dep }} \leqslant 220 \mathrm{~K}$, as shown in Fig. 3(b). Taking into account that one molecule is able to host several clusters after deposition at low temperature and that some nodes remain uncovered at higher temperature, clusters on the nodes have about twice the size of the clusters on the phenyl rings, as illustrated in the models in Fig. 3. We note that a continuous transition of the cluster nucleation site from on top of the organic molecules to the coordination nodes takes place upon coverage increase at low deposition temperature. For Fe coverages about six times higher than the one of Fig. 2(b) on (NC-Ph $4-\mathrm{CN})_{3} \mathrm{Co}_{2}$, nearly every node is occupied by one Fe cluster. From this we conclude that the (a)

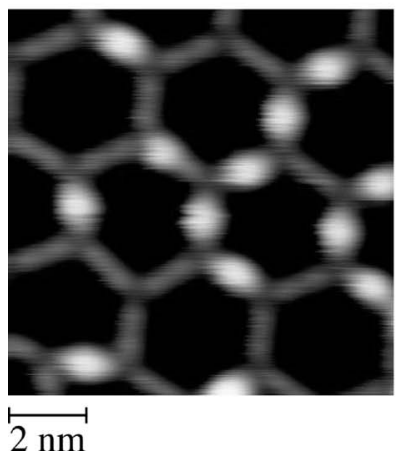

(b)

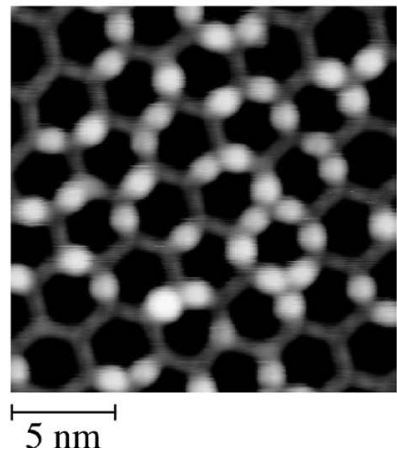

(c)

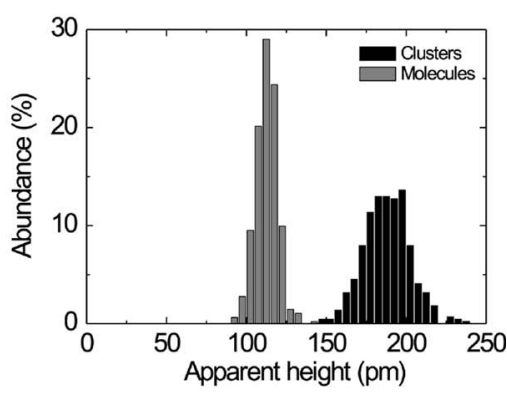

FIG. 2. (a) Constant current STM image of the Co decorated $\left(\mathrm{NC}-\mathrm{Ph}_{3}-\mathrm{CN}\right)_{3} \mathrm{Co}_{2}$ network $\left[\right.$ deposition temperature $T_{\text {dep }}=116 \mathrm{~K}$, coverage $\Theta=0.018 \pm 0.006$ ML, one monolayer (ML) is defined as one atom per $\mathrm{Ag}(111)$ unit cell, $I_{t}=0.06 \mathrm{nA}$, and $V_{t}=-0.5 \mathrm{~V}$ (Ref. 28)]. (b) STM image of the Fe decorated $\left(\mathrm{NC}-\mathrm{Ph}_{3}-\mathrm{CN}\right)_{3} \mathrm{Co}_{2}$ network $\left(T_{\mathrm{dep}}=120 \mathrm{~K}, \Theta=0.027 \pm 0.017 \mathrm{ML}, I_{t}=0.1 \mathrm{nA}\right.$, and $\left.V_{t}=-0.5 V\right)$. (c) Histogram of apparent heights of molecules (gray) and of Fe clusters (black) with respect to the $\mathrm{Ag}(111)$ surface. The histogram has been derived from a large scale STM image recorded for the same deposition conditions as (b). 
(a)

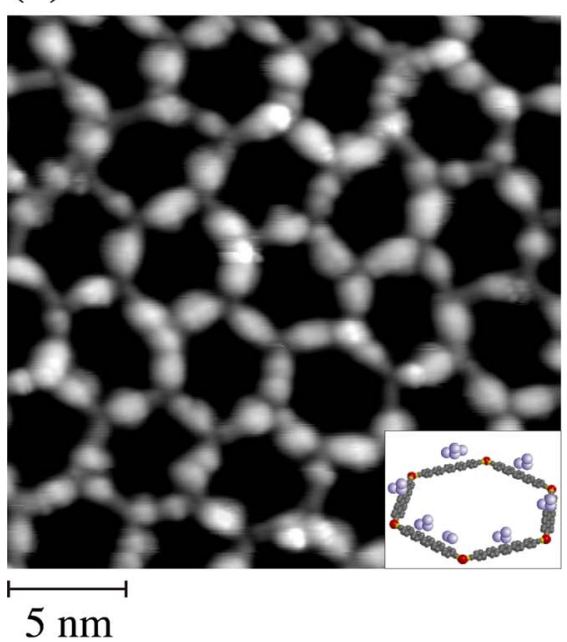

(b)

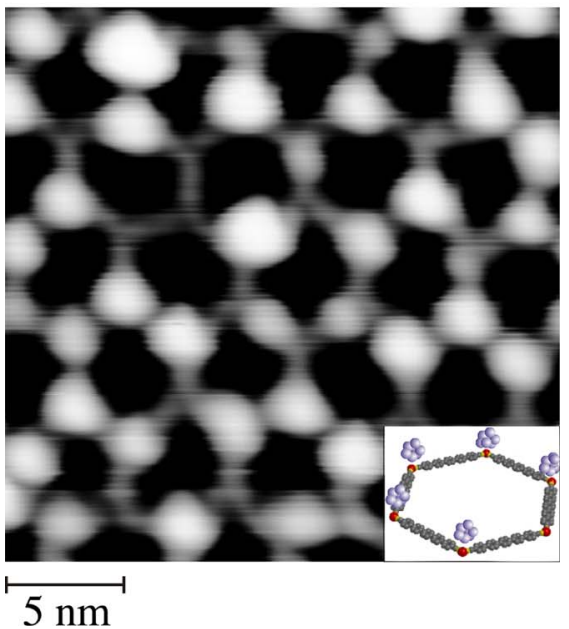

FIG. 3. (Color online) Selective decoration of organic molecules (a) or Co coordination nodes (b) depending on Fe deposition temperature. Template $\left(\mathrm{NC}-\mathrm{Ph}_{5}-\mathrm{CN}\right)_{3} \mathrm{Co}_{2} . \quad$ (a) $T_{\text {dep }}=130 \mathrm{~K} \quad\left(I_{t}=0.1 \mathrm{nA}, V_{t}=-0.5 \mathrm{~V}\right)$. (b) $T_{\text {dep }}$ $=190 \mathrm{~K}\left(I_{t}=0.1 \mathrm{nA}, V_{t}=-1.0 \mathrm{~V}\right)$. The insets schematically show the position of the Fe clusters. $\Theta=0.054 \pm 0.030 \mathrm{ML}$ for both figures.

organic molecules are metastable, and the coordination nodes stable nucleation sites.

The Fe or Co decorated networks are stable up to about $250 \mathrm{~K}$. Beyond this temperature the Fe or Co decorated templates start to disintegrate, although the undecorated metalorganic networks are stable up to room temperature.

In conclusion, we have shown the use of metal-organic $\left(\mathrm{NC}-\mathrm{Ph}_{n}-\mathrm{CN}\right)_{3} \mathrm{Co}_{2}$ honeycomb networks with a tunable lattice constant as templates to steer the location and with it the chemical environment, as well as the shape and spacing of small $\mathrm{Fe}$ and Co clusters. The preferential nucleation sites are the organic network ligands for deposition temperatures from $90 \mathrm{~K}$ to $130 \mathrm{~K}$, while $\mathrm{Fe}$ deposition at around $200 \mathrm{~K}$ leads to nucleation at the network nodes. In both cases, regular nanopatterns of magnetic atoms are formed. The clusters assembled on top of the metal-organic network are likely decoupled from the substrate and interactions are only pos- sible via the metal-organic template. This is expected to give rise to interesting electronic and magnetic properties.

We gratefully acknowledge financial support from the Swiss National Science Foundation and from the European Science Foundation Collaborative Research Program FunSMARTs.

${ }^{1}$ P. Gambardella, S. Rusponi, M. Veronese, S. S. Dhesi, C. Grazioli, A. Dallmeyer, I. Cabria, R. Zeller, P. H. Dederichs, K. Kern, C. Carbone, and H. Brune, Science 300, 1130 (2003).

${ }^{2}$ M. Haruta, Catal. Today 36, 153 (1997).

${ }^{3}$ U. Heiz and W. D. Schneider, Crit. Rev. Solid State Mater. Sci. 26, 251 (2001).

${ }^{4}$ D. D. Chambliss, R. J. Wilson, and S. Chiang, Phys. Rev. Lett. 66, 1721 (1991).

${ }^{5}$ H. Brune, M. Giovannini, K. Bromann, and K. Kern, Nature (London) 394, 451 (1998).

${ }^{6}$ H. Brune, Surf. Sci. Rep. 31, 121 (1998).

${ }^{7}$ A. N'Diaye, S. Bleikamp, P. J. Feibelman, and T. Michely, Phys. Rev. Lett. 97, 215501 (2006).

${ }^{8}$ V. Repain, G. Baudot, H. Ellmer, and S. Rousset, Europhys. Lett. 58, 730 (2002).

${ }^{9}$ C. Didiot, S. Pons, B. Kierren, Y. Fagot-Revurat, and D. Malterre, Nat. Nanotechnol. 2, 617 (2007).

${ }^{10}$ N. Weiss, T. Cren, M. Epple, S. Rusponi, G. Baudot, S. Rohart, A. Tejeda, V. Repain, S. Rousset, P. Ohresser, F. Scheurer, P. Bencok, and H. Brune, Phys. Rev. Lett. 95, 157204 (2005).

${ }^{11}$ M. Schmid, G. Kresse, A. Buchsbaum, E. Napetschnig, S. Gritschneder, M. Reichling, and P. Varga, Phys. Rev. Lett. 99, 196104 (2007).

${ }^{12}$ S. Degen, C. Becker, and K. Wandelt, Faraday Discuss. 125, 343 (2004).

${ }^{13}$ J. V. Barth, Annu. Rev. Phys. Chem. 58, 375 (2007).

${ }^{14}$ S. Stepanow, M. Lingenfelder, A. Dmitriev, H. Spillmann, E. Delvigne, N. Lin, X. Deng, C. Cai, J. V. Barth, and K. Kern, Nature Mater. 3, 229 (2004)

${ }^{15}$ J. A. Theobald, N. S. Oxtoby, M. A. Phillips, N. R. Champness, and P. H. Beton, Nature (London) 424, 1029 (2003).

${ }^{16}$ X. Ma, H. L. Meyerheim, J. Barthel, J. Kirschner, S. Schmitt, and E. Umbach, Appl. Phys. Lett. 84, 4038 (2004).

${ }^{17}$ U. Schlickum, R. Decker, F. Klappenberger, G. Zoppellaro, S. Klyatskaya, M. Ruben, I. Silanes, A. Arnau, K. Kern, H. Brune, and J. V. Barth, Nano Lett. 7, 3813 (2007).

${ }^{18}$ S. Clair, S. Pons, A. P. Seitsonen, H. Brune, K. Kern, and J. V. Barth, J. Phys. Chem. B 108, 14585 (2004).

${ }^{19}$ U. Schlickum, R. Decker, F. Klappenberger, G. Zoppellaro, S. Klyatskaya, W. Auwärter, S. Neppl, K. Kern, H. Brune, M. Ruben, and J. V. Barth, J. Am. Chem. Soc. 130, 11778 (2008).

${ }^{20}$ S. Stepanow, N. Lin, D. Payer, U. Schlickum, F. Klappenberger, G. Zoppellaro, M. Ruben, H. Brune, J. V. Barth, and K. Kern, Angew. Chem., Int. Ed. 46, 710 (2007).

${ }^{21}$ A. Schiffrin, J. Reichert, W. Auwärter, G. Jahnz, Y. Pennec, A. WeberBargioni, V. S. Stepanyuk, L. Niebergall, P. Bruno, and J. V. Barth, Phys. Rev. B 78, 035424 (2008).

${ }^{22}$ C. Eschenbroich and A. Salzer, Organometallchemie (B. G. Teubner, Stuttgart, 1990).

${ }^{23}$ F. Meyer, F. A. Khan, and P. B. Armentrout, J. Am. Chem. Soc. 117, 9740 (1995).

${ }^{24}$ J. J. BelBruno, Surf. Sci. 577, 167 (2005).

${ }^{25}$ P. W. Davies, M. A. Quinlan, and G. A. Somorjai, Surf. Sci. 121, 290 (1982).

${ }^{26}$ G. Boisvert, L. J. Lewis, M. J. Puska, and R. M. Nieminen, Phys. Rev. B 52, 9078 (1995).

${ }^{27}$ I. Horcas, R. Fernández, J. M. Gómez-Rodríguez, J. Colchero, J. GómezHerrero, and A. M. Baro, Rev. Sci. Instrum. 78, 013705 (2007).

${ }^{28}$ For negative bias voltages the apparent height of the ligands is smaller than for positive ones, while the imaging of the clusters is largely independent of bias voltage, therefore contrast is maximized for negative bias. 